\title{
Dementia and delirium, the outcomes in elderly hip fracture patients
}

\author{
This article was published in the following Dove Press journal: \\ Clinical Interventions in Aging \\ 10 March 2017 \\ Number of times this article has been viewed
}

\author{
Christina A Mosk' \\ Marnix Mus' \\ Jos PAM Vroemen' \\ Tjeerd van der Ploeg ${ }^{2}$ \\ Dagmar I Vos' \\ Leon HGJ Elmans ${ }^{3}$ \\ Lijckle van der Laan' \\ 'Department of Surgery, Amphia \\ Hospital, Breda, ${ }^{2}$ Department of \\ Public Health, Erasmus MC-University \\ Medical Center, Rotterdam, \\ ${ }^{3}$ Department of Orthopedic \\ Surgery, Amphia Hospital, Breda, \\ the Netherlands
}

Background: Delirium in hip fractured patients is a frequent complication. Dementia is an important risk factor for delirium and is common in frail elderly. This study aimed to extend the previous knowledge on risk factors for delirium and the consequences. Special attention was given to patients with dementia and delirium.

Methods: This is a retrospective cohort study performed in the Amphia Hospital, Breda, the Netherlands. A full electronic patient file system (Hyperspace Version IU4: Epic, Inc., Verona, WI, USA) was used to assess data between January 2014 and September 2015. All patients presented were aged $\geq 70$ years with a hip fracture, who underwent surgery with osteosynthesis or arthroplasty. Patients were excluded in case of a pathological or a periprosthetic hip fracture, multiple traumatic injuries, and high-energy trauma. Patient and surgical characteristics were documented. Postoperative outcomes were noted. Delirium was screened using Delirium Observation Screening Scale and dementia was assessed from medical notes.

Results: Of a total of 566 included patients, $75 \%$ were females. The median age was 84 years (interquartile range: 9). Delirium was observed in 35\%. Significant risk factors for delirium were a high American Society of Anesthesiology score, delirium in medical history, functional dependency, preoperative institutionalization, low hemoglobin level, and high amount of blood transfusion. Delirium was correlated with a longer hospital stay $(P=0.001)$, increased association with complications $(P<0.001)$, institutionalization $(P<0.001)$, and 6-month mortality $(P<0.001)$. Patients with dementia $(\mathrm{N}=168)$ had a higher delirium rate $(57.7 \%, P<0.001)$ but a shorter hospital stay $(P<0.001)$. There was no significant difference in the 6-month mortality between delirious patients with (34.0\%) and without dementia (26.3\%).

Conclusion: Elderly patients with a hip fracture are vulnerable for delirium, especially when the patient has dementia. Patients who underwent an episode of delirium were at increased risk for adverse outcomes.

Keywords: hip fracture, elderly, dementia, delirium, complications, adverse outcomes

\section{Introduction}

Hip fractures are an important cause of hospitalization in elderly people and, even those with major comorbidities, are in need of surgical intervention. The number of persons aged $>70$ will double within the next three decades, which will result in an increase in the incidence of hip fractures. ${ }^{1}$

More than any other type of fracture, a hip fracture in elderly is associated with loss of independence, high morbidity and mortality (36\%), and high health care costs. ${ }^{2}$

A major complication in elderly hip fracture patients is delirium, with an incidence rate varying from $13 \%$ to $70 \% .{ }^{3-6}$ Postoperative delirium is associated with poor outcomes, such as impaired functional and cognitive recovery, increased hospital length of stay, higher costs, and increased mortality. ${ }^{3-12}$
Correspondence: Christina A Mosk Department of Surgery, Amphia Hospital, 905I8, 4800 RK Breda, the Netherlands

Tel +3I 765952653

Email amosk@amphia.nl 
No strong evidence exists on the treatment of delirium. ${ }^{13,14}$ However, Inouye et $\mathrm{al}^{8}$ stated that in the general geriatric population, $30 \%$ to $40 \%$ of the delirium episodes could be prevented by treatment of the risk factors. Several other studies were performed on delirium prevention in hip fracture patients, but the evidence remains weak. ${ }^{14-16}$ Orthogeriatric care for example has been described as an effective treatment for this patient group, but delirium was not always taken into account or the evidence was weak. ${ }^{17,18}$ Furthermore, a diversity of risk factors has been described in a heterogeneous group of patients and studies. Most studies were conducted on frail elderly, cardiothoracic surgery patients, patients admitted to long-term facility care or intensive care unit (ICU). ${ }^{19-22}$

Dementia is one of the most important risk factors for delirium in the elderly and its prevalence is increasing. ${ }^{23}$ The effects of dementia on the outcome after delirium (delirium superimposed on dementia) have been rarely described and, to the best of our knowledge, never in elderly hip fracture patients. ${ }^{24,25}$

The goal of this study was to add to the previous knowledge on the risk factors for delirium in elderly hip fracture patients. In addition, this study investigated the effects of dementia on delirium and the outcomes after an episode of delirium in this patient category.

\section{Patient and methods Patient selection}

Ethical approval was deemed unnecessary by the institutional review board of the Amphia hospital due to its retrospective nature. Written consent was also deemed unnecessary by the institutional review board. Patients aged $\geq 70$ years with an isolated hip fracture were included between January 1, 2014 and August 31, 2015 at the Amphia Hospital, Breda, the Netherlands. Patients included in this study underwent hip replacement therapy or osteosynthesis. In the event of a second hip fracture during the course of this study, data related to only the first hip fracture was used.

A hip fracture was defined as proximal femur fracture and classified according to the Arbeitsgemeinschaft für Osteosynthesefragen (AO) classification: $31 \mathrm{~A}$ or $31 \mathrm{~B}$. Femoral head fractures (AO $31 \mathrm{C}$ ) were not included.

Patients with a pathological or a peri-prosthetic hip fracture, as well as patients with multiple traumatic injuries and high-energy trauma were excluded.

The follow-up was documented by physicians and registered nurses. All study parameters were collected in an electronic patient file system: Hyperspace Version IU4
(Epic, Inc., Verona, WI, USA). Data were collected in validated scoring systems used in the electronic patient file, eg, Katz Index of Independence in Activities of Daily Living (Katz-ADL), Short Nutritional Assessment Questionnaire for Residential Care, Mini Nutritional Assessment Short-Form, or Delirium Observational Screening Scale (DOSS) screening score. All data were analyzed retrospectively.

The follow-up was 6 months or until death. Date of mortality was retrieved from the CompeT\&T database (Eindhoven, the Netherlands).

\section{Preoperative data}

Patient characteristics, sex, age, comorbidity, and factors of frailty were documented. Burden of comorbidity was assessed by the Charlson comorbidity index (CCI) and American Society of Anesthesiology (ASA) physical status classification. ${ }^{26,27}$

The CCI categorizes comorbidities and weighs them from 0 to 6 , with 6 having the highest chance for mortality. Age is also included with 1 point for each decade since the age of 50 . The sum of all the factors results in the CCI score. ${ }^{28}$

The presence of the following comorbidities was registered, these were grouped as follows: 1) previous hip fracture; 2) presence of cardiac morbidity (eg, arrhythmia, myocardial infarction, heart failure, valve insufficiency or stenosis, acute coronary syndrome, and coronary artery bypass graft); 3) pulmonary morbidity (eg, chronic obstructive pulmonary disease, emphysema, tuberculosis, asthma, lung cancer, and lung embolus); 4) neurological morbidity (eg, cerebrovascular accident or transient ischemic attack, epilepsy, Parkinson, meningioma, subarachnoid bleeding); 5) renal insufficiency; 6) previous delirium and dementia; 7) presence of diabetes; and 8) osteoporosis. Also the use of anticoagulants was registered. The duration of admission until surgical intervention was noted. ${ }^{29}$

\section{Factors of frailty}

Nutritional status was assessed using the Short Nutritional Assessment Questionnaire for Residential Care score, which is based on the body mass index, loss of weight and reduced appetite, and need of help with eating. A cut-off of 3 points or more was used to indicate severe undernourishment. ${ }^{30}$

Physical impairment was evaluated using the Katz-ADL score. The Katz-ADL scores physical performances: bathing, dressing, toileting, transfers, feeding, and continence. Less than 6 points indicated functional impairment and $<2$ points indicated severe functional impairment. ${ }^{31}$ 
Social status and living arrangement, use of alcohol, vision, and hearing problems were scored.

\section{Treatment}

The following procedures were performed for hip fracture repair: inter- or subtrochanteric fractures (AO 31 A types) were repaired by a gamma nail (Stryker Corporation ${ }^{\circledR}$, Kalamazoo, MI, USA) or a dynamic hip screw (Depuy-Synthes ${ }^{\circledR}$, PA, USA).

Intracapsular fractures (AO 31 B types) were treated with either osteosynthesis (dynamic hip screw, a dynamic locking blade plate (Gannet ${ }^{\circledR}$, Groningen, the Netherlands) or DepuySynthes cannulated screws) or hemi- or total arthroplasty.

Information was collected concerning the type of surgery, type of anesthesia, duration of anesthesia (intubation and extubation time), blood loss (mL), and events which occurred during surgery. All the patients had received preoperative prophylactic antibiotics.

\section{Primary outcome}

The DOSS was used to screen for postoperative delirium (POD). DOSS were taken daily during each nurse shift. A DOSS $\geq 3$, during three or more nurse working shifts in a row, was considered as highly specific for delirium and a criterion to consult a geriatrician. This was also done in case there was any doubt about the diagnosis, and in patients with dementia. For the current study, the clinical notes of the geriatric department were analyzed to confirm the diagnosis of delirium.

Delirium duration was defined as the number of days with DOSS $\geq 3.32,33$ If a patient was discharged with high DOSS ( $\geq 3$ ), the duration of the delirium was counted until the day of discharge.

Use of prophylactic or therapeutic medication (eg, haloperidol, lorazepam, or seroquel) was started by protocol and noted. The physician could deviate from the protocol.

\section{Other postoperative outcomes}

Adverse events were divided into surgery-related events (eg, wound infection, postoperative bleeding, hardware failure of open reduction internal fixation material or prosthetic luxation after hip replacement and a new hip fracture within 6 months) and medical events (eg, cardiac, urinary, neurological, or pulmonary).

Data were gathered on ICU admission, the duration of hospital stay (excluding in-hospital mortality patients), anemia, and the amount of administered packed cells. Packed cells were indicated when hemoglobin level was $<5 \mathrm{mmol} / \mathrm{L}$ $(8 \mathrm{~g} / \mathrm{dL})$ in patients $>60$ years in the general population and $<6 \mathrm{mmol} / \mathrm{L}(9.7 \mathrm{~g} / \mathrm{dL})$ in patients with cardiac conditions or symptomatic anemia. ${ }^{34}$

Mortality within 6 months after surgery, location of discharge (home with or without care or institutionalization) was described.

\section{Statistical analysis}

Frequencies and descriptive statistics were used for reporting the baseline characteristics of the variables in our study population.

Differences in categorical variables between patients with or without delirium were presented as frequencies with percentages and tested using the chi-square test or Fisher's exact test. The Kolmogorov-Smirnov test was used to define normal distribution for continuous variables. Differences in continuous variables were presented as medians (interquartile ranges [IQR]) or means (standard deviation) and were tested using the Mann-Whitney $U$-test or Student's $t$-test, respectively.

Length of delirium with respect to medication was compared using the Kruskal-Wallis test.

To perform a univariate risk analysis of risk factors related to the incidence of delirium, odds ratios with $95 \%$ confidence interval were used. Multivariate analysis was done, using logistic regression to correct for confounder (if the rule of thumb was not violated). All significant risk factors were adjusted for age. Survival rates were calculated using Kaplan-Meier analysis, and differences with respect to subgroups were analyzed using the Log-rank test.

A $P$-value $<0.05$ was considered significant. Statistical analysis was performed with SPSS Version 23.0 (SPSS Inc., Chicago, IL, USA) software.

\section{Results}

\section{Baseline clinical characteristics}

Table 1 shows all baseline preoperative characteristics. Data of 566 included patients were extracted from the electronic patient files. Of these patients, 425 patients (75\%) were females and the median age was 84 years (IQR: 9). The ASA score of 3 was most often documented (364 patients, $63.3 \%$ ) and the median CCI was 6 (IQR: 2 ). The majority of the patients had an intracapsular fracture (58\%).

In 506 patients (89.4\%), surgery occurred within 24 hours of admission. Median duration of hospital stay in the total patient group was 6 days (IQR: 3 ).

\section{Delirium}

A total of 196 patients (35\%) had a delirium during hospital stay and follow-up. One patient was re-admitted within 
Table I Baseline characteristics of the study population

\begin{tabular}{|c|c|c|}
\hline Patient characteristics & Total $\mathbf{N}=566$ & $\%$ \\
\hline \multicolumn{3}{|l|}{ Sex } \\
\hline Female & 425 & 75.0 \\
\hline Male & $|4|$ & 25.0 \\
\hline Age, Median (IQR) & $84(9)$ & \\
\hline CCI, Median (IQR) & $6(2)$ & \\
\hline \multicolumn{3}{|l|}{ ASA score } \\
\hline I & 12 & 2.1 \\
\hline 2 & 159 & 27.8 \\
\hline 3 & 364 & 63.6 \\
\hline 4 & 31 & 5.5 \\
\hline \multicolumn{3}{|l|}{ Medical history } \\
\hline Contralateral hip fracture & 45 & 8.0 \\
\hline Cardial & 248 & 44.0 \\
\hline Pulmonal (COPD) & 107 & 18.9 \\
\hline Neurological ${ }^{\mathrm{a}}$ & 203 & 35.9 \\
\hline Renal insufficiency & 82 & 14.5 \\
\hline Delirium in medical history & 139 & 24.8 \\
\hline Dementia & 168 & 29.7 \\
\hline Diabetes & 133 & 23.5 \\
\hline Osteoporosis & 95 & 16.8 \\
\hline BMI, Median (IQR) & $23.6(5.1)$ & \\
\hline SNAQ-RC & $0.54(1.2)$ & \\
\hline 0 & 425 & 74.3 \\
\hline $\mathrm{I}-2$ & 52 & 9.2 \\
\hline$\geq 3$ & 63 & 11.1 \\
\hline Katz-ADL, Median (IQR) & $5(3)$ & \\
\hline Daily use of alcohol & 116 & 20.5 \\
\hline Visual impairment & 319 & 56.3 \\
\hline Hearing impairment & 223 & 39.4 \\
\hline Preoperative $\mathrm{Hb}$ level (mmol/L), Median (IQR) & $7.7(1.4)$ & \\
\hline Nursing home prior to admission & 173 & 30.6 \\
\hline Help at home prior to admission & 130 & 23.0 \\
\hline \multicolumn{3}{|l|}{ Fracture type } \\
\hline Intracapsular & 329 & 58.0 \\
\hline Intertrochanteric & 206 & 36.4 \\
\hline Subtrochanteric & 31 & 5.5 \\
\hline
\end{tabular}

Note: axcluding dementia and delirium.

Abbreviations: ASA, American Society of Anesthesiology; BMI, body mass index; $\mathrm{CCl}$, Charlson Comorbidity score; Hb, hemoglobin; Katz-ADL, Katz Index of Independence in Activities of Daily Living; COPD, chronic obstructive pulmonary disease; IQR, interquartile range; SD, standard deviation; SNAQ-RC, Short Nutritional Assessment Questionnaire for Residential Care.

1 week after discharge to the geriatric ward with delirium. Within the delirious group, 146 patients were women (74\%). The median age was 85.5 (IQR: 8 ), $P<0.001$. Preoperatively, 46 patients were delirious. In the majority of the patients with POD (112 patients, $74.7 \%$ ), delirium started 1 day after surgery. The median delirium duration was 4 days (IQR: 4) (Table 2).

\section{Risk factors for delirium}

Logistic regression was permitted because the rule of thumb was not violated (ten variables were found, and there were 196 events of delirium).
The following risk factors were significantly associated with incidence of delirium on univariate and multivariate analyses, respectively (adjusted for age): age (OR: 2.65 [1.71-4.10]), CCI (1.17 [1.06-1.30]), and ASA score (2.47 [1.62-3.75]), (0.45[0.30-0.70]); the presence of dementia $(0.25[0.16-0.35]),(0.26[0.18-0.38])$; a previous delirium episode (0.29 [0.20-0.44]), (0.31 [0.21-0.46]); functional dependency (Katz-RC) (0.23 [0.16-0.34]), (0.26 [0.18$0.40])$; postoperative hemoglobin level (0.77 [0.66-0.92]), (0.83 [0.70-0.98]); the amount of transfusions (1.23 [1.06-1.43]), (1.20 [1.03-1.40]); home care prior to admission $(0.23$ [0.15-0.33]), (0.26 [0.17-0.38]); institutionalization prior to admission $(0.28[0.10-0.41]),(0.31[0.21-0.45])$. Table 2 summarizes the risk factors for delirium.

The amount of packed cell transfusions in the delirious group ranged from 0 to 8 packed cells (median: 0, IQR: 1), whereas the amount of blood transfusions in the nondelirious group ranged from 0 to 6 packed cells (median: 0, IQR: 1), $P=0.041$.

\section{Perioperative risk factors}

Osteosynthesis was performed in 266 cases (47\%): a gamma nail in 173 patients (30.6\%), a dynamic hip screw in 63 patients (11.1\%), a Gannet in 18 patients (3.2\%), and three Cancellous screws 12 patients (2.1\%).

A total of 300 patients (53\%) underwent prosthetic repair, of which 35 underwent total arthroplasty (6.2\%) and 265 hemi-arthroplasty (47\%).

No significant difference was found between the incidence of delirium and the use of either osteosynthesis or arthroplasty $(P=0.738$; Table 2$)$.

\section{Postoperative outcome}

The following postoperative outcome were significantly correlated with delirium: longer length of hospital stay (median 6 days [IQR: 6], $P=0.002$ ), higher rate of complications (94 patients, $48.5 \%, P<0.001$ ) and higher incidence of mortality within 6 months (59 patients, $30.1 \%, P<0.001$ ).

A significantly higher rate of patients with a delirium were care-dependent and admitted to a nursing home (180 patients, $91.8 \%, P<0.001$ ). Table 3 shows the outcomes after delirium. Mortality is shown in the Kaplan-Meier curve (Figure 1).

\section{Delirium and dementia}

A total of 168 patients (30\%) had a form of dementia at admission. One hundred twenty-two patients (73\%) had dementia, DOSS $\geq 3$ during three work shifts in a row was 
Table 2 Risk factors for delirium

\begin{tabular}{|c|c|c|c|c|c|c|}
\hline \multirow[t]{2}{*}{ Delirium outcomes } & \multirow{2}{*}{$\frac{\text { No delirium }}{(\mathrm{N}=370)(65.4 \%)}$} & \multirow{2}{*}{$\begin{array}{l}\text { Delirium } \\
(\mathrm{N}=196)(34.6 \%)\end{array}$} & \multirow{2}{*}{$\frac{\text { Total }}{\mathrm{N}=566}$} & \multirow[t]{2}{*}{$P$-value } & \multirow{2}{*}{$\begin{array}{l}\text { (uni) Odds } \\
\text { ratio (Cl 95\%) }\end{array}$} & \multirow{2}{*}{$\begin{array}{l}\text { Adjusted odds } \\
\text { ratio (Cl 95\%) }\end{array}$} \\
\hline & & & & & & \\
\hline Preoperative delirium & $0(0)$ & $46(23.5)$ & 46 & & & \\
\hline Duration of delirium (days), Median (IQR) & $0(0)$ & $4(4)$ & $4(4)$ & & & \\
\hline Delirium at discharge & $0(0)$ & $100(5 \mathrm{I})$ & 100 & & & \\
\hline Died while delirious & $0(0)$ & $8(4)$ & 8 & & & \\
\hline \multicolumn{7}{|l|}{ Risk factors } \\
\hline Sex & & & & & $1.05(0.7 \mathrm{I}-\mathrm{I} .60)$ & \\
\hline Female (ref) & $279(75.4)$ & $146(74.5)$ & 425 & & & \\
\hline Male & $91(24.6)$ & $50(25.5)$ & 141 & 0.811 & & \\
\hline Age & & & & & $2.65(1.7 \mid-4.10)$ & \\
\hline $70-79$ & $126(34.0)$ & $32(16.3)$ & 158 & & & \\
\hline $80+($ ref) & $244(66.0)$ & I $64(83.7)$ & 408 & $<0.001$ & & \\
\hline Median (IQR) & $83(10)$ & $85.5(8)$ & & $<0.00 \mathrm{I}^{\wedge}$ & $1.07(1.04-1.10)$ & \\
\hline \multicolumn{7}{|l|}{ Burden of comorbidity } \\
\hline CCl, Median (IQR) & $5.0(3)$ & $6.0(2)$ & & $<0.001^{\wedge}$ & $1.17(1.06-1.30)$ & $1.09(0.98-1.21)$ \\
\hline \multicolumn{7}{|l|}{ ASA } \\
\hline $\mathrm{I}-2$ & $132(35.7)$ & $36(18.4)$ & 168 & & & \\
\hline $3-4$ (ref) & $238(64.3)$ & $160(81.6)$ & 398 & $<0.001$ & $2.47(1.62-3.75)$ & $0.45(0.30-0.70)^{\mathrm{c}}$ \\
\hline \multicolumn{7}{|l|}{ Medical history } \\
\hline Contralat hip fracture & $28(7.6)$ & $17(8.7)$ & 45 & 0.644 & $1.16(0.62-2.20)$ & \\
\hline Cardial & $164(44.3)$ & $85(43.4)$ & 248 & 0.827 & $0.96(0.68-1.36)$ & \\
\hline Pulmonal (COPD) & $68(18.4)$ & $39(20.0)$ & 107 & 0.660 & $1.10(0.7|-| .7 I)$ & \\
\hline Neurological ${ }^{\mathrm{a}}$ & $127(34.3)$ & $76(38.7)$ & 203 & 0.293 & $1.21(0.85-1.74)$ & \\
\hline Renal insufficiency & $49(13.2)$ & $33(16.8)$ & 82 & 0.248 & $1.33(0.82-2.14)$ & \\
\hline Dementia & 71 (19.2) & $97(49.5)$ & 168 & $<0.001$ & $0.25(0.16-0.35)$ & $0.26(0.18-0.38)^{c}$ \\
\hline Previous delirium & $61(16.5)$ & $78(40.0)$ & 139 & $<0.001$ & $0.29(0.20-0.44)$ & $0.31(0.21-0.46)^{c}$ \\
\hline Missing data (\%) & & & $6(1.1)$ & & & \\
\hline Diabetes & $82(22.2)$ & $5 I(26.0)$ & 133 & 0.303 & I.24 (0.83-I.85) & \\
\hline Osteoporosis & $70(18.9)$ & $25(12.7)$ & 95 & 0.062 & $0.63(0.38-1.03)$ & \\
\hline BMI, Median (IQR) & $23.6(4.8)$ & $23.5(5.8)$ & & $0.561^{\wedge}$ & $0.99(0.95-1.04)$ & \\
\hline SNAQ $<3$ & $317(85.7)$ & $160(81.6)$ & 479 & & & \\
\hline SNAQ $\geq 3$ (ref) & $36(10.1)$ & $25(12.7)$ & 61 & 0.240 & $1.39(0.80-2.34)$ & \\
\hline Daily alcohol use & $79(21.3)$ & 37 (18.9) & 116 & 0.503 & $0.86(0.56-1.33)$ & \\
\hline Visual impairment & $203(55)$ & $116(59.2)$ & 319 & 0.325 & $1.20(0.84-1.70)$ & \\
\hline Hearing impairment & $137(37)$ & $86(43.9)$ & 223 & 0.113 & $1.33(0.93-1.90)$ & \\
\hline \multicolumn{7}{|l|}{ Functional dependency } \\
\hline Katz $\leq$ score 5 & I56 (42.I) & I $46(75.0)$ & 302 & $<0.001$ & $0.23(0.16-0.34)$ & $0.26(0.18-0.40)^{c}$ \\
\hline Katz $\leq$ score 2 & $55(14.9)$ & $83(42.3)$ & 138 & $<0.001$ & $0.23(0.15-0.34)$ & $0.24(0.16-0.40)^{c}$ \\
\hline Missing data (\%) & & & $8(1.4)$ & & & \\
\hline \multicolumn{7}{|l|}{ AO classification } \\
\hline $3|a|$ (ref) & $54(14.6)$ & $35(17.9)$ & 89 & 0.666 & & \\
\hline $31 \mathrm{a} 2$ & $62(16.8)$ & $33(16.8)$ & 95 & & $0.8(0.5-1.5)$ & \\
\hline $31 \mathrm{a} 3$ & $30(8.1)$ & $19(10.0)$ & 49 & & $0.9(0.4-1.8)$ & \\
\hline $3|b|$ & $53(14.3)$ & $21(10.7)$ & 74 & & $0.6(0.3-1.2)$ & \\
\hline $3 \mathrm{lb} 2$ & $81(22.0)$ & $37(19.0)$ & 118 & & $0.7(0.4-1.3)$ & \\
\hline $3 \mathrm{lb3}$ & $90(24.3)$ & $51(26.0)$ & $14 \mid$ & & $0.8(0.5-1.5)$ & \\
\hline Preoperative anticoagulants & $201(54.3)$ & $105(53.6)$ & 306 & 0.864 & $0.97(0.69-1.40)$ & \\
\hline Kind of anesthesia & & & & 0.393 & $0.82(0.53-1.28)$ & \\
\hline General (ref) & 292 (78.9) & $161(82.1)$ & 453 & & & \\
\hline Epidural & $77(20.8)$ & $35(17.8)$ & 112 & & & \\
\hline Duration of surgery minutes, Median (IQR) & $77(24)$ & $69(23)$ & & $0.455^{\wedge}$ & 1.00 & \\
\hline Blood loss cc, Median (IQR) & $200(200)$ & $200(150)$ & & $0.564^{\wedge}$ & $1.00(0.99-1.00)$ & \\
\hline \multicolumn{7}{|l|}{ Duration of admission until surgery } \\
\hline$<24$ hours (ref) & $130(35.1)$ & $62(31.6)$ & 192 & 0.696 & $1.16(0.801 .70)$ & \\
\hline $24-48$ hours & $202(54.6)$ & $112(57.1)$ & 314 & & & \\
\hline$>48$ hours & $38(10.3)$ & $22(I I . I)$ & 60 & & & \\
\hline Operation description & & & & 0.738 & $0.94(0.66-1.33)$ & \\
\hline Osteosynthesis (ref) & $172(46.5)$ & $94(35.3)$ & 266 & & & \\
\hline Arthroplasty & $198(53.5)$ & $102(34.0)$ & 300 & & & \\
\hline
\end{tabular}


Table 2 (Continued)

\begin{tabular}{|c|c|c|c|c|c|c|}
\hline \multirow[t]{2}{*}{ Delirium outcomes } & \multirow{2}{*}{$\frac{\text { No delirium }}{(\mathbf{N}=370)(65.4 \%)}$} & \multirow{2}{*}{$\begin{array}{l}\text { Delirium } \\
(N=196)(34.6 \%)\end{array}$} & \multirow{2}{*}{$\frac{\text { Total }}{N=566}$} & \multirow[t]{2}{*}{$P$-value } & \multirow{2}{*}{$\begin{array}{l}\text { (uni) Odds } \\
\text { ratio (Cl 95\%) }\end{array}$} & \multirow{2}{*}{$\begin{array}{l}\text { Adjusted odds } \\
\text { ratio (Cl 95\%) }\end{array}$} \\
\hline & & & & & & \\
\hline \multicolumn{7}{|l|}{ Hemoglobin level (mmol/L) } \\
\hline Preoperative Hb level, Median (IQR) & $7.8(1.3)$ & $7.6(1.3)$ & & $0.05 I^{\wedge}$ & $0.86(0.73-1.02)$ & \\
\hline Postoperative Hb level, Median (IQR) & $6.6(1.5)$ & $6.2(1.6)$ & & $0.002^{\wedge}$ & $0.77(0.66-0.92)$ & $0.83(0.70-0.98)^{c}$ \\
\hline Missing data $(\%)$ & & & $10(1.7)$ & & & \\
\hline Blood transfused & $99(26.8)$ & 66 (33.7) & 165 & 0.085 & $1.40(0.95-2.02)$ & \\
\hline Amount, Median (IQR) & $0(I)$ & $0(I)$ & & $\left.0.04\right|^{\wedge}$ & $1.23(1.06-1.43)$ & $1.20(1.03-1.40)^{c}$ \\
\hline \multicolumn{7}{|l|}{ Home care prior to admission } \\
\hline Care prior to admission & I54 (4I.6) & $149(76.0)$ & 303 & $<0.001$ & $0.23(0.15-0.33)$ & $0.26(0.17-0.38)^{c}$ \\
\hline Nursing home & $78(2 I . I)$ & $96(48.5)$ & 174 & $<0.001$ & $0.28(0.10-0.41)$ & $0.31(0.21-0.45)^{c}$ \\
\hline Care at home & $76(20.8)$ & $53(27.0)$ & 129 & 0.079 & $0.71(0.47-1.06)$ & \\
\hline \multicolumn{7}{|l|}{ Medication use $\mathrm{e}^{\mathrm{b}}$} \\
\hline No medication (ref) & $303(82.0)$ & $22(11.2)$ & 325 & $<0.001$ & & \\
\hline Prophylactic & $62(16.7)$ & $71(36.2)$ & 133 & & $0.06(0.04-0.11)$ & \\
\hline Therapeutic & $5(1.3)$ & $103(52.6)$ & 108 & & I $8.00(6.88-47.0)$ & \\
\hline
\end{tabular}

Notes: Log regression for univariate measurements; Cl 95\%; adjusted OR for age. Katz $\leq$ score 5 physical impairment, Katz $\leq$ score 2 severe physical impairment. ${ }^{a}$ Excluding dementia and delirium. ${ }^{b} \mathrm{Haloperidol,} \mathrm{seroquel,} \mathrm{lorazepam.}{ }^{~ S i g n i f i c a n t ~ a s s o c i a t i o n ~ w i t h ~ d e l i r i u m ~ w h e n ~ a d j u s t e d ~ f o r ~ a g e ; ~ P e a r s o n ~ c h i-s q u a r e ~ t e s t ; ~}{ }^{\wedge}$ Mann-Whitney $U$-test; IQR: Interquartile range (of the median), missing data described in case of missing data and significant outcome.

Abbreviations: ASA, American Society of Anesthesiology; BMI, body mass index; cc, cubic centimeter; Cl, confidence interval; CCl, Charlson comorbidity score; COPD, chronic obstructive pulmonary disease; Hb, hemoglobin; IQR, interquartile range; Katz, Katz Index of Independence; OR, odds ratio; SNAQ, Short Nutritional Assessment Questionnaire; uni, univariate odds ratio.

described, presupposing a delirium. Geriatric consultation confirmed delirium in $97(57.7 \%)$ of the 168 patients with dementia.

Preoperatively, 27 patients with dementia (16.1\%) were delirious versus 19 patients $(4.8 \%)$ without dementia, $P<0.001$. Hence, the incidence of postoperative delirium was $35 \%$ (70 patients) in the patient group with dementia versus $21 \%$ (80 patients) in the group without dementia, $P<0.001$.

The specific outcomes in patients with dementia and with a delirium are described in Table 4.

\section{Discussion}

The aim of this study was to add to the knowledge about risk factors of delirium and outcome after a delirium in elderly hip fracture patients who underwent acute surgical intervention. In this study, special attention was given to delirium superimposed on dementia.

Our study revealed a delirium incidence of $34.3 \%$. The important risk factors appeared to be old age, high burden of comorbidity, dementia, previous delirium, functional dependency, low postoperative hemoglobin level, and high number of blood transfusions. Postoperatively, delirium was correlated with a higher incidence of complications, longer length of hospital stay, higher functional dependency, and a higher rate of mortality within 6 months. As $57.7 \%$ of the patients with dementia suffered from delirium, dementia appeared to be one of the most important risk factors for delirium. Patients with dementia and with delirium had a shorter admission duration and were more often institutionalized. Furthermore, a trend toward a higher incidence of 6-month mortality after an episode of delirium was noted.

The observed incidence of delirium in our study population (34\%) and specifically in patients with dementia (58\%) is in line with previous studies. ${ }^{8,25,35,36}$ Inouye et al described that delirium in the elderly population occurred in $13 \%-55 \%$, with a higher percentage in surgical patients, especially hip fracture patients. ${ }^{8}$ Fick et al described $32 \%$ delirium in the specific group of patients with dementia. However, this was in nonsurgical patients. ${ }^{25}$ Striking is the fact that 46 of the 196 patients were delirious before surgery. In these cases, prevention of delirium was not possible.

The risk factors shown in our study that correlate with delirium should be assessed at admission to prevent delirium. Several risk factors in the current study are in line with previous research. ${ }^{8,37,38}$ Former studies used the ASA scale, as well as the CCI or Acute Physiology and Chronic Health Evaluation score. A high score, correlating with severe comorbidities, was often found to be significantly related to POD. ${ }^{35}$ In the current study, a high score on the ASA scale and CCI is also reported as significantly related to POD. ${ }^{22,35,39}$

Functional dependency was confirmed as a significant risk factor for delirium. ${ }^{35,37}$ However, Oh et al described that after multivariate analyses, functional impairment was not significantly correlated with delirium. ${ }^{35}$ These studies all used other measurements to define functional impairment 
Table 3 Outcomes in delirious patients versus patients without a delirium

\begin{tabular}{|c|c|c|c|c|}
\hline \multirow[t]{2}{*}{ Postoperative outcomes } & \multirow{2}{*}{$\begin{array}{l}\text { No delirium } \\
\mathrm{N}=370(65.4 \%)\end{array}$} & \multirow{2}{*}{$\begin{array}{l}\text { Delirium } \\
\mathrm{N}=196(34.6 \%)\end{array}$} & \multirow{2}{*}{$\begin{array}{l}\text { Total } \\
N=566\end{array}$} & \multirow[t]{2}{*}{$P$-value } \\
\hline & & & & \\
\hline Length of ICU admission, Median (IQR) & $0(0)$ & $0(0)$ & & $0.657^{\wedge}$ \\
\hline ICU admission & $8(2.1)$ & $6(3.0)$ & 14 & $0.573^{*}$ \\
\hline $\mathrm{ICU} \geq 2$ days & $2(0.5)$ & $2(1.0)$ & 4 & $0.612^{*}$ \\
\hline Number of patients with a complication & $93(25.1)$ & $94(48.0)$ & 187 & $<0.001$ \\
\hline Contralateral hip fracture within 6 months & $0(0)$ & $4(2.0)$ & 4 & $0.014^{*}$ \\
\hline Luxation & $5(1.4)$ & $\mathrm{I}(0.5)$ & 6 & $0.670^{*}$ \\
\hline OSM outbreak & $5(1.4)$ & $4(2.0)$ & 9 & $0.505^{*}$ \\
\hline Cardiac complication & $7(1.8)$ & $9(4.6)$ & 16 & 0.065 \\
\hline Pulmonary complication & $10(2.7)$ & $17(8.7)$ & 27 & 0.002 \\
\hline Neurological ${ }^{a}$ & $4(I . I)$ & $7(3.6)$ & 10 & $0.037^{*}$ \\
\hline Renal function disorders & $6(1.6)$ & $13(6.6)$ & 19 & 0.002 \\
\hline Urinary tract infection & $14(3.8)$ & $23(117)$ & 37 & $<0.001$ \\
\hline Bladder retention & $15(4.0)$ & $17(8.7)$ & 32 & 0.024 \\
\hline Infection & $\mathrm{I}(0.3)$ & $2(1.0)$ & 3 & $0.276^{*}$ \\
\hline Postoperative bleeding & II (2.9) & $10(5.1)$ & 21 & 0.202 \\
\hline Other complications ${ }^{\mathrm{b}}$ & $25(6.7)$ & $30(15.3)$ & 55 & 0.001 \\
\hline Hemodynamic instability & $6(1.6)$ & $10(5.1)$ & 16 & 0.017 \\
\hline Pneumonia & $6(1.6)$ & $12(6.1)$ & 18 & 0.004 \\
\hline Myocardial infarction & $2(0.5)$ & $\mathrm{I}(0.5)$ & 3 & $1.000^{*}$ \\
\hline Cardiac decompensation & $6(1.6)$ & $6(3.0)$ & 12 & $0.258^{*}$ \\
\hline Cardiac arrhythmia (AF) & $4(1.0)$ & $6(3.0)$ & 10 & $0.102 *$ \\
\hline CVA/TIA & $3(0.8)$ & $7(3.5)$ & 10 & $0.037^{*}$ \\
\hline Nerve damage & $\mathrm{I}(0.3)$ & I (0.5) & 2 & $1.000 *$ \\
\hline Length of hospital stay, Median (IQR) & $5(3)$ & $6(6)$ & & $0.00 I^{\wedge}$ \\
\hline \multicolumn{5}{|l|}{ Mortality } \\
\hline In-hospital mortality & $4(\mathrm{I} . \mathrm{I})$ & $7(3.6)$ & 11 & $0.055^{*}$ \\
\hline$<6$-month mortality ${ }^{\mathrm{c}}$ & $38(10.4)$ & $52(27.5)$ & 90 & $<0.001$ \\
\hline Total mortality $<6$ months & $42(11.4)$ & $59(30.1)$ & 101 & $<0.001$ \\
\hline \multicolumn{5}{|l|}{ Care after discharge } \\
\hline Same residence & $|5|(40.8)$ & $95(48.5)$ & 246 & 0.080 \\
\hline New residence & $215(58.1)$ & $94(48.0)$ & 309 & 0.021 \\
\hline Home without care & $26(7.0)$ & I (0.5) & 27 & 0.001 \\
\hline Home with care & $54(14.6)$ & $8(4.1)$ & 62 & $<0.001$ \\
\hline Nursing home ${ }^{d}$ & $286(77.3)$ & $180(91.8)$ & 466 & $<0.001$ \\
\hline
\end{tabular}

Notes: Pearson chi-square test; *Fisher's Exact Test; ^Mann-Whitney U-test. 'Excluding delirium. 'Decubitus, ileus, scrotal abscess, electrolyte abnormalities, abdominal complaints, irregular glucoses values, iatrogenic complications during admission. 'Excluding in-hospital mortality. ${ }^{\mathrm{d}}$ Revalidation, nursing home, or hospice.

Abbreviations: CVA/TIA, cerebrovascular accident or transient ischemic attack; ICU, intensive care unit; IQR, interquartile range; AF, atrial fibrillation; OSM, osteosyntheses material.

or dependency. In the current study, functional dependency was described using the Katz-ADL. Patients often needed home care or were institutionalized prior to admission. In our opinion, these three findings are correlated.

In this study, a low postoperative hemoglobin ( $\mathrm{Hb}$ ) level was shown to be a risk factor for delirium. A low $\mathrm{Hb}$ level has not been associated with delirium in previous studies. However, former studies did describe a significantly higher incidence of delirium in elderly hospitalized anemic patients. ${ }^{40,41}$ This could be explained by the fact that after surgery, blood loss causes lower Hb levels but not necessarily anemia. In addition, the previous studies did not specifically describe the postoperative $\mathrm{Hb}$ levels, only whether a patient was anemic or not.
The daily consumption of alcohol, visual and hearing impairment are often described as risk factors for delirium. ${ }^{8,39}$ This was not confirmed in our study but is often described in general medicine.

This study also concluded that POD was highly correlated with short-term complications and long-term adverse outcomes. These outcomes are consistent with former research. ${ }^{8,22,24,42}$

The POD was significantly associated with pulmonary and cardiac complications, urinary tract infections and bladder retention, ICU admission, length of hospital stay, functional dependency, and mortality. ${ }^{42,43}$ Besides comorbidity and the surgical procedure, these postoperative complications could have caused the delirious episode. The question 
Table 4 Delirium superimposed on dementia. Incidence and outcome

\begin{tabular}{|c|c|c|c|}
\hline \multirow[t]{2}{*}{ Postoperative outcomes } & \multirow{2}{*}{$\begin{array}{l}\text { No dementia } \\
\mathrm{N}=398(\%)\end{array}$} & \multirow{2}{*}{$\begin{array}{l}\text { Dementia } \\
N=168(\%)\end{array}$} & \multirow[t]{2}{*}{$P$-value } \\
\hline & & & \\
\hline Delirium $(n=196)$ & $99(25.0)$ & $97(57.7)$ & $<0.001$ \\
\hline Preoperative delirium & $19(4.8)$ & $27(16.1)$ & $<0.001$ \\
\hline \multirow[t]{3}{*}{ Length of delirium, Median (IQR) } & $4(4)$ & $3(4)$ & $0.718^{\wedge}$ \\
\hline & Delirium without & DSD; & \\
\hline & dementia; $\mathbf{N}=99$ (\%) & $\mathbf{N}=97(\%)$ & \\
\hline Number of patients with $\geq$ one complications & $57(57.6)$ & $37(38.1)$ & 0.006 \\
\hline Cardiac complication & $6(6.0)$ & $3(3.1)$ & $0.498^{*}$ \\
\hline Pulmonary complication & $13(13.1)$ & $4(4.1)$ & 0.025 \\
\hline Neurological $^{\mathrm{a}}$ & $5(5.1)$ & $3(3.1)$ & $0.721^{*}$ \\
\hline Renal function disorders & $8(8.1)$ & $5(5.1)$ & 0.410 \\
\hline Urinary tract infection & $13(13.1)$ & $10(10.3)$ & 0.539 \\
\hline Bladder retention & II (II.I) & $6(6.2)$ & 0.221 \\
\hline Other complications ${ }^{\mathrm{b}}$ & $20(20.2)$ & $10(10.3)$ & 0.054 \\
\hline Hemodynamic instability & $6(6.1)$ & $4(4.1)$ & $0.748^{*}$ \\
\hline Pneumonia & $9(9.1)$ & $3(3.1)$ & 0.080 \\
\hline Myocardial infarction & $I(1.0)$ & $0(0)$ & $1.000^{*}$ \\
\hline Cardiac decompensation & $4(4.0)$ & $2(2.0)$ & $0.683^{*}$ \\
\hline Cardiac rhythm disorder & $2(2.0)$ & $4(4.1)$ & $0.442^{*}$ \\
\hline CVA/TIA & $3(3.0)$ & $4(4.1)$ & $0.719 *$ \\
\hline Length of hospital stay, Median (IQR) & $7(6)$ & $4(4)$ & $<0.001^{\wedge}$ \\
\hline New residence & $64(64.6)$ & $29(29.9)$ & $<0.001$ \\
\hline Nursing home & $86(88)$ & $92(94.8)$ & 0.053 \\
\hline In-hospital mortality & $5(5.1)$ & $2(2.1)$ & $0.445^{*}$ \\
\hline$<6$ month mortalityc & $21(22.3)$ & $31(32.6)$ & 0.113 \\
\hline Total mortality $<6$ months & $26(26.3)$ & $33(34.0)$ & 0.236 \\
\hline
\end{tabular}

Notes: ${ }^{a}$ Excluding delirium. ${ }^{b}$ Decubitus, ileus, scrotal abscess, electrolyte abnormalities, abdominal complaints, irregular glucose values, iatrogenic complications during admission. 'Excluding in-hospital mortality; Pearson chi square test; *Fisher's Exact Test; ^Mann-Whitney U-test.

Abbreviations: CVA/TIA, cerebrovascular accident or transient ischemic attack; DSD, delirium superimposed on dementia; IQR, interquartile range of the median.

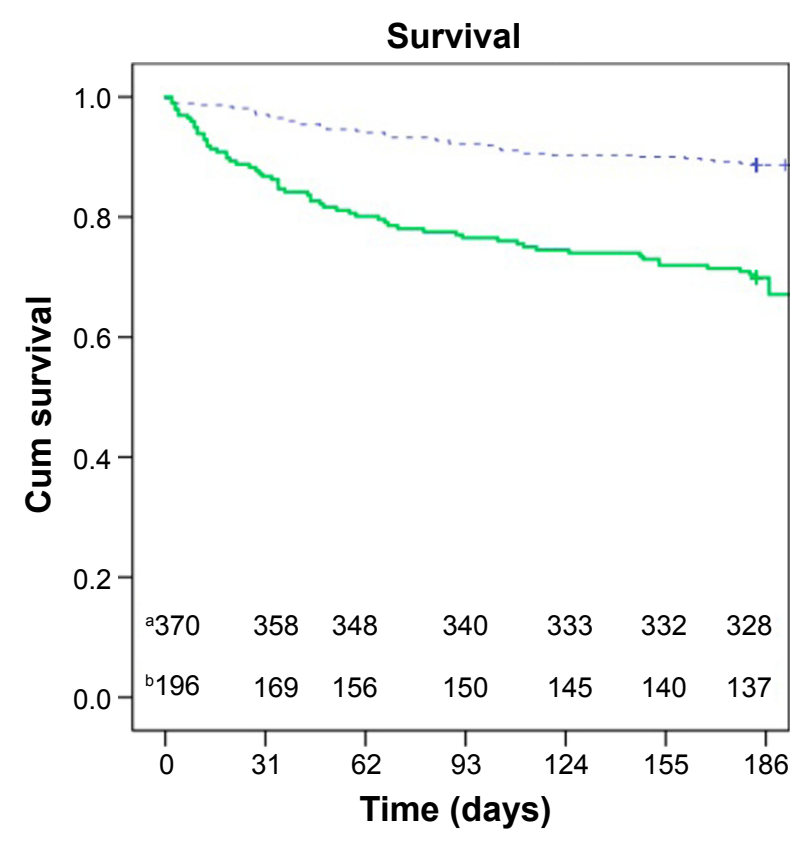

\begin{tabular}{|l}
\hline No delirium \\
+ 0-censored
\end{tabular}

Figure I Overall survival within 6 months. Delirium versus no delirium.

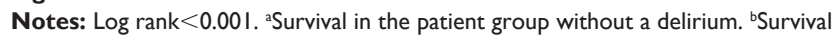
in the patient group with a delirium.

Abbreviation: cum, cumulative. remains which occurred first, the episode of delirium or the other postoperative complications. ${ }^{7,8}$

It was expected that patients with dementia and with delirium would have worse outcome compared to nondemented patients. However, this was not confirmed in this study.

The non-demented patient group with a delirium had a significantly higher complication rate, a higher chance to be admitted to a new nursery home and a longer length of stay. Patients with dementia often quickly return to their previous nursery home and therefore have a shorter length of stay and a lower chance for other complications during hospital stay.

As described before, delirium, in general, has important short- and long-term adverse outcomes. Patients with dementia are vulnerable, due to their functional dependency and (often) poor nutritional state. This could lead to the expectation that the outcomes would be worse than the outcomes of nondemented patients. ${ }^{44}$ In addition, former research has clearly described the impact of delirium in patients with dementia and found that these patients required a longer hospital stay and showed greater functional impairment. ${ }^{25}$ However, functional impairment was described by Katz$\mathrm{ADL}$ at follow-up, whereas our study described functional 
dependency using Katz-ADL and the need for care or institutionalization at admission and discharge. These outcomes found in our study are explained by the fact that most patients with dementia already were institutionalized and discharged early back to their nursing homes. By contrast, patients who were not demented and became delirious had to be admitted to a new nursing home for rehabilitation. Waiting lists for rehabilitation care in our setting are long, which explains a longer hospital stay and the increased risk for complications.

Mortality within 6 months was higher in delirious patients than in nondelirious patients, which is in line with former research. ${ }^{9,11}$ However, Dolan et al who also described an increased risk for mortality in delirious patients, described that after adjustment for confounders, delirium was no longer significantly associated with mortality. ${ }^{10}$

The mortality rate in patients with dementia did not significantly differ from patients without dementia, which is in line with former research of Fick et al and McCusker et al. ${ }^{25,45}$ However, both studies were conducted in nonsurgical wards. The present article is one of a few to describe outcomes after delirium, with specific attention to patients with dementia, ie, delirium superimposed on dementia.

\section{Limitations and strength}

This study has several limitations. This study was set up retrospectively and therefore not all predictors for delirium could be gathered and some were incomplete. Cognitive impairment or dementia was evaluated using medical notes. No distinction was made between severity of cognitive impairment. In addition, dementia often presents itself at admission, which might result in an underestimation of the number of patients with dementia in this study. Unfortunately, in our clinic informedbased interview like Informant Questionnaire on Cognitive Decline in the Elderly was not used to determine dementia. DOSS is officially a validated instrument for screening delirium and does not diagnose whether a patient is delirious, but it has a high sensitivity of $97 \% .^{33,46}$ DOSS has never been validated for patients with dementia, but geriatricians have diagnosed delirium in this patient category. ${ }^{32,47}$

The strength of this study is that a great number of patients are investigated. The electronic patient file, which was implemented in 2013, was used to gather all data. In addition, also patients with dementia were included, which was often an exclusion criterion in other studies. . $^{8,10,11,25}$

\section{Conclusion}

Our results demonstrate the need for: 1) improvement of preventive care for delirium; 2) specific attention to patients with dementia for frailty and prevention of delirium; and 3) early diagnosis and treatment of delirium and its underlying causes.

It is crucial to identify and validate as many risk factors as possible to create a risk model for vulnerable patients of each specific patient group. In addition, more research is needed to define risk factors for delirium and to obtain knowledge on the outcomes after delirium, especially in patients with dementia.

\section{Disclosure}

This research is funded by an "unrestricted grant" by the Amphia fund for innovation. The author reports no conflicts of interest in this work.

\section{References}

1. World Health Organization. Ageing and health. Available from: http://www.who.int/mediacentre/factsheets/fs404/en/ 2015. Accessed September 1, 2015.

2. Carpintero P, Caeiro JR, Carpintero R, Morales A, Silva S, Mesa M. Complications of hip fractures: a review. World J Orthop. 2014;5(4): 402-411.

3. Bruce AJ, Ritchie CW, Blizard R, Lai R, Raven P. The incidence of delirium associated with orthopedic surgery: a meta-analytic review. Int Psychogeriatr. 2007;19(2):197-214.

4. Krogseth M, Watne LO, Juliebo V, et al. Delirium is a risk factor for further cognitive decline in cognitively impaired hip fracture patients. Arch Gerontol Geriatr. 2016;64:38-44.

5. Krogseth M, Wyller TB, Engedal K, Juliebo V. Delirium is a risk factor for institutionalization and functional decline in older hip fracture patients. J Psychosom Res. 2014;76(1):68-74.

6. Vochteloo AJ, Borger van der Burg BL, Tuinebreijer WE, et al. Do clinical characteristics and outcome in nonagenarians with a hip fracture differ from younger patients? Geriatr Gerontol Int. 2013;13(1):190-197.

7. Gleason LJ, Schmitt EM, Kosar CM, et al. Effect of delirium and other major complications on outcomes after elective surgery in older adults. JAMA Surg. 2015;150(12):1134-1140.

8. Inouye SK, Westendorp RG, Saczynski JS. Delirium in elderly people. Lancet (London, England). 2014;383(9920):911-922.

9. Bellelli G, Mazzola P, Morandi A, et al. Duration of postoperative delirium is an independent predictor of 6-month mortality in older adults after hip fracture. J Am Geriatr Soc. 2014;62(7):1335-1340.

10. Dolan MM, Hawkes WG, Zimmerman SI, et al. Delirium on hospital admission in aged hip fracture patients: prediction of mortality and 2-year functional outcomes. J Gerontol A Biol Sci Med Sci. 2000; 55(9): M527-M534.

11. Kat MG, de Jonghe JF, Vreeswijk R, et al. Mortality associated with delirium after hip-surgery: a 2-year follow-up study. Age Ageing. 2011;40(3): 312-318.

12. Zywiel MG, Hurley RT, Perruccio AV, Hancock-Howard RL, Coyte PC, Rampersaud YR. Health economic implications of perioperative delirium in older patients after surgery for a fragility hip fracture. J Bone Joint Surg Am. 2015;97(10):829-836.

13. Schrijver EJ, de Graaf K, de Vries OJ, Maier AB, Nanayakkara PW. Efficacy and safety of haloperidol for in-hospital delirium prevention and treatment: a systematic review of current evidence. Eur J Intern Med. 2016;27:14-23.

14. Siddiqi N, Harrison JK, Clegg A, et al. Interventions for preventing delirium in hospitalised non-ICU patients. Cochrane Database Syst Rev. 2016;3:CD005563.

15. Inouye SK, Bogardus ST Jr, Charpentier PA, et al. A multicomponent intervention to prevent delirium in hospitalized older patients. $N \mathrm{Engl}$ J Med. 1999;340(9):669-676. 
16. Marcantonio ER, Flacker JM, Wright RJ, Resnick NM. Reducing delirium after hip fracture: a randomized trial. J Am Geriatr Soc. 2001; 49(5):516-522.

17. Kammerlander C, Roth T, Friedman SM, et al. Ortho-geriatric service - a literature review comparing different models. Osteoporos Int. 2010;21(Suppl 4):S637-S646.

18. Prestmo A, Hagen G, Sletvold O, et al. Comprehensive geriatric care for patients with hip fractures: a prospective, randomised, controlled trial. Lancet. 2015;385(9978):1623-1633.

19. Bilotta F, Lauretta MP, Borozdina A, Mizikov VM, Rosa G. Postoperative delirium: risk factors, diagnosis and perioperative care. Minerva Anestesiol. 2013;79(9):1066-1076.

20. Inouye SK. Predisposing and precipitating factors for delirium in hospitalized older patients. Dement Geriatr Cogn Disord. 1999; 10(5):393-400.

21. Rahkonen T, Eloniemi-Sulkava U, Halonen P, et al. Delirium in the non-demented oldest old in the general population: risk factors and prognosis. Int J Geriatr Psychiatry. 2001;16(4):415-421.

22. Robinson TN, Raeburn CD, Tran ZV, Angles EM, Brenner LA, Moss M. Postoperative delirium in the elderly: risk factors and outcomes. Ann Surg. 2009;249(1):173-178.

23. Alzheimer's A. 2015 Alzheimer's disease facts and figures. Alzheimers Dement. 2015;11(3):332-384.

24. Fong TG, Davis D, Growdon ME, Albuquerque A, Inouye SK. The interface between delirium and dementia in elderly adults. Lancet Neurol. 2015;14(8):823-832.

25. Fick DM, Steis MR, Waller JL, Inouye SK. Delirium superimposed on dementia is associated with prolonged length of stay and poor outcomes in hospitalized older adults. J Hosp Med. 2013;8(9):500-505.

26. Charlson ME, Pompei P, Ales KL, MacKenzie CR. A new method of classifying prognostic comorbidity in longitudinal studies: development and validation. J Chronic Dis. 1987;40(5):373-383.

27. Owens WD, Felts JA, Spitznagel EL Jr. ASA physical status classifications: a study of consistency of ratings. Anesthesiology. 1978; 49(4):239-243.

28. Deyo RA, Cherkin DC, Ciol MA. Adapting a clinical comorbidity index for use with ICD-9-CM administrative databases. J Clin Epidemiol. 1992;45(6):613-619.

29. Bretherton CP, Parker MJ. Early surgery for patients with a fracture of the hip decreases 30-day mortality. Bone Joint J. 2015;97$\mathrm{B}(1): 104-108$.

30. Kruizenga HM, de Vet HC, Van Marissing CM, et al. The SNAQ(RC), an easy traffic light system as a first step in the recognition of undernutrition in residential care. J Nutr Health Aging. 2010;14(2):83-89.

31. Wallace M, Shelkey M, Hartford Institute for Geriatric N. Katz Index of Independence in Activities of Daily Living (ADL). Urol Nurs. 2007;27(1):93-94.

32. Schuurmans MJ, Shortridge-Baggett LM, Duursma SA. The Delirium Observation Screening Scale: a screening instrument for delirium. Res Theory Nurs Pract. 2003;17(1):31-50.
33. Raats JW, van Eijsden WA, Crolla RM, Steyerberg EW, van der Laan L. Risk factors and outcomes for postoperative delirium after major surgery in elderly patients. PLoS One. 2015;10(8):e0136071.

34. Haas FJLM. dVRRP. Guideline blood transfusion. Tijdschrift Bloedtransfusie. 2011;4:102-105.

35. Oh ES, Li M, Fafowora TM, et al. Preoperative risk factors for postoperative delirium following hip fracture repair: a systematic review. Int J Geriatr Psychiatry. 2015;30(9):900-910.

36. Yang Y, Zhao X, Dong T, Yang Z, Zhang Q, Zhang Y. Risk factors for postoperative delirium following hip fracture repair in elderly patients: a systematic review and meta-analysis. Aging Clin Exp Res. Epub 2016 Feb 12.

37. Ahmed S, Leurent B, Sampson EL. Risk factors for incident delirium among older people in acute hospital medical units: a systematic review and meta-analysis. Age Ageing. 2014;43(3):326-333.

38. Scholz AF, Oldroyd C, McCarthy K, Quinn TJ, Hewitt J. Systematic review and meta-analysis of risk factors for postoperative delirium among older patients undergoing gastrointestinal surgery. Br J Surg. 2016;103(2): e21-e28.

39. Kalisvaart KJ, Vreeswijk R, de Jonghe JF, van der Ploeg T, van Gool WA, Eikelenboom P. Risk factors and prediction of postoperative delirium in elderly hip-surgery patients: implementation and validation of a medical risk factor model. J Am Geriatr Soc. 2006;54(5):817-822.

40. Joosten E, Hiele M, Ghoos Y, Pelemans W, Boogaerts MA. Diagnosis of iron-deficiency anemia in a hospitalized geriatric population. Am J Med. 1991;90(5):653-654.

41. Vochteloo AJ, Borger van der Burg BL, Mertens B, et al. Outcome in hip fracture patients related to anemia at admission and allogeneic blood transfusion: an analysis of 1262 surgically treated patients. BMC Musculoskelet Disord. 2011;12:262.

42. Witlox J, Eurelings LS, de Jonghe JF, Kalisvaart KJ, Eikelenboom P, van Gool WA. Delirium in elderly patients and the risk of postdischarge mortality, institutionalization, and dementia: a meta-analysis. JAMA. 2010;304(4):443-451.

43. Robinson TN, Eiseman B. Postoperative delirium in the elderly: diagnosis and management. Clin Interv Aging. 2008;3(2):351-355.

44. Volkert D, Chourdakis M, Faxen-Irving G, et al. ESPEN guidelines on nutrition in dementia. Clin Nutr. 2015;34(6):1052-1073.

45. McCusker J, Cole MG, Voyer P, et al. Six-month outcomes of cooccurring delirium, depression, and dementia in long-term care. J Am Geriatr Soc. 2014;62(12):2296-2302.

46. Gemert van LA, Schuurmans MJ. The Neecham Confusion Scale and the Delirium Observation Screening Scale: capacity to discriminate and ease of use in clinical practice. BMC Nurs. 2007;6:3.

47. Scheffer AC, van Munster BC, Schuurmans MJ, de Rooij SE. Assessing severity of delirium by the Delirium Observation Screening Scale. Int J Geriatr Psychiatry. 2011;26(3):284-291.
Clinical Interventions in Aging

\section{Publish your work in this journal}

Clinical Interventions in Aging is an international, peer-reviewed journal focusing on evidence-based reports on the value or lack thereof of treatments intended to prevent or delay the onset of maladaptive correlates of aging in human beings. This journal is indexed on PubMed Central, MedLine,

\section{Dovepress}

CAS, Scopus and the Elsevier Bibliographic databases. The manuscript management system is completely online and includes a very quick and fair peer-review system, which is all easy to use. Visit http://www.dovepress. com/testimonials.php to read real quotes from published authors. 